\title{
A constrained filtering algorithm for freeway traffic state estimation
}

\author{
Mariano A. Risso( $\left.{ }^{*}\right)^{1,2}$, Neila Bhouri ${ }^{4}$, Aldo J. Rubiales ${ }^{1,3}$ and Pablo A. Lotito ${ }^{1,2}$ \\ PLADEMA, Universidad Nacional del Centro de la Prov. de Buenos Aires, Argentina ${ }^{1}$ \\ CONICET - Consejo Nacional de Investigaciones Científicas y Técnicas, Argentina ${ }^{2}$ \\ CICPBA - Consejo de Investigaciones Científicas Buenos Aires, Argentina ${ }^{3}$ \\ Université Paris Est, IFSTTAR, GRETTIA, France. ${ }^{4}$
}

September 15, 2017

\begin{abstract}
A real-time traffic state estimation algorithm is developed and applied to a freeway. The evolution of the traffic is defined by a second order macroscopic model which computes, for each section of the freeway, the density and the mean speed according to several non linear equations. Different extensions of the Kalman method were already applied to this model, though none of them considers the natural constraints in the state variables. In this work, a new method that incorporates those natural constraints, is applied to the macroscopic model obtaining better results. To validate the proposed method, a simulation over a freeway section was made using two different tools: the macroscopic simulator called METANET and the microscopic simulator called SUMO. Promising results were obtained using both approaches.

Keywords: Freeway; Traffic state estimation; Kalman filter;

Corresponding(*) author. Tel.: +54-249-4385690.

E-mail address: mrisso@exa.unicen.edu.ar
\end{abstract}

\section{Introduction}

Traffic congestion is a major problem around the world. Traffic optimization tools and Intelligent Transport Systems (ITS) are designed to provide real-time control and route guidance for drivers and to optimize the performance of traffic networks (Stanciu et al., 2012). Several methods and approaches were developed to accomplish this task. In freeway traffic, intelligent vehicle freeway systems (IVHS) that distribute intelligence between roadside infrastructure and vehicles are one of the most promising solutions to the traffic congestion problem. The IVHS must be fed with real-time information provided by several sensors that are disseminated all over the traffic network and measure diverse aspects of the traffic flow. Obtaining an accurate and complete state of the traffic network concerns the State Estimation field. Vehicular Traffic state estimation for a freeway network amounts to estimate the value of the traffic state variables with the information provided from many sources. More precisely, as it is stated in (Papageorgiou and Blosseville, 1990, Papageorgiou et al., 1990), based on some directly measured traffic variables, the estimation algorithm should calculate and complete all the traffic variables associated to each element of the traffic network for a given time.

An extensive account of the research on Traffic state estimation is made in the recent work (Seo et al., 2017). Following the definitions given in that work, the method presented here is a Data Assimilation approach. These methods are based on the design of a dynamical system whose trajectory approach the desired target quantity. The typical example is the Kalman filter (Simon, 2010) which is the best option when the dynamics are linear, unconstrained and with Gaussian errors. The history of this kind of methods can be dated back to (Szeto and Gazis, 1972) where the authors develop an extended Kalman filter to 
estimate the density and to obtain a control law, using the now called Fundamental Diagram for the (non linear) observation equation.

Traffic is known to have a highly non-linear behavior (Treiber and Helbing, 2002) with many vehicle interactions what makes the issue of estimation by means of aggregated variables absolutely challenging. Macroscopic models may be used to describe such behavior, representing the traffic average behavior in terms of aggregated variables (flow, density and average speed) (Hoogendoorn and Bovy, 2001). The macroscopic models in a freeway are classified as first order, second order or superior order, considering the number of differential (or difference) equations included.

In (Bhouri et al., 1989) with a very simple first order linear model the authors arrive to estimate the density using a scalar Kalman filter over a section of a freeway. Later on, other works considered the first order model called CTM (Cell Transmission Model) (Daganzo (1994) and Daganzo (1995)). CTM models the density behavior in a freeway link, each link is divided into homogeneous sections (cells) whose length is related to the distance traveled by a vehicle at free speed in one time step. Then, from the flow conservation equation at each segment, a single equation for the evolution of the density is obtained. This equation is non linear because it involves the minimum operator.

In (Mihaylova and Boel, 2004) and (Mihaylova et al., 2006) the evolution of the traffic is modeled as a dynamic stochastic system based on CTM. While in the first paper a Particle Filter is used as estimation method, in the second the Unscented Kalman Filter (UKF) is used. A comparison of both methods is depicted in (Mihaylova et al., 2007) and a parallelization of the particle filter version is presented in (Hegiy et al., 2007).

In (Munoz et al., 2003, Alvarez-Icaza et al., 2004) the SMM model, a piecewise-affine extension of the CTM model, is presented and used to predict traffic density based on boundary measures. More recently, in (Canudas-De-Wit et al., 2012), traffic density is estimated using a dynamic observer for the SMM with inputs given by the measurements at the boundaries of the network. This model is also used in (Vivas et al., 2016, Brandi et al., 2017), where distributed consensus-based switched observers are proposed for the density estimation in a traffic system.

In (Marinica and Boel, 2009) a particle filter method (PF) is also applied to the CTM model; however, it is used to estimate urban traffic. The traffic network considered in this paper consists in signaled intersections, the streets that join these intersections, and vehicle flow sensors. PF assigns weights to each particle considering the rightness of the signals of the sensor which is observed and generates estimations at each time of the location of the bottlenecks. It also estimates the length of the queues at each intersection.

As it is stated in (Gomes and Horowitz, 2006), first order models lead to simpler formulations and optimization problems but they are not able to reproduce the capacity drop (observed difference between the freeway capacity and the queue discharge rate) in contrast to second-order models that have this advantage.

The second order model (Messner and Papageorgiou, 1990) is a discretized and enhanced variation of the Payne model (Payne, 1971). It also considers that the freeway is divided into consecutively numbered sections, each one with a respective length and a number of lanes. For each section, the model calculates the density, the flow and the average speed according to several equations which accurately model the merging and lane drop phenomena (Spiliopoulou et al., 2014).

In (Wang and Papageorgiou, 2005) extended Kalman filter (EKF) method uses the second-order macroscopic model to freeway traffic state estimation. An algorithm based on the UKF version of the Kalman filter and on the previously mentioned model (Messner and Papageorgiou, 1990) is presented in (Ngoduy, 2011) showing good results. However, the UKF method can fail when the variables are restricted, see the survey (Simon, 2010). Furthermore, in the case of the traffic model studied here, negative speed or density values could be obtained, as it is shown in this paper at the end of section 3 .

The traffic flow of a freeway stretch belonging to the northern of Beijing city in China is estimated in (Zhang et al., 2013). In this work a particle filter algorithm based on the second-order macroscopic traffic flow model proposed by Papageorgiou (Papageorgiou et al., 1990) is applied. 


\section{Considering that}

- the second order model represents in a more realistic manner all the freeway traffic conditions (Gomes and Horowitz, 2006),

- the EKF was applied successfully to this model in (Wang and Papageorgiou, 2005),

- there are UKF variants that improve the results of the EKF method as it is demonstrated in other areas(Zhang and Xia, 2011, Risso et al., 2015),

- standard UKF algorithm can give estimations outside the variable domain which make the algorithm to stop (Teixeira et al., 2008),

the goal of this work is to develop a constrained filtering method that combines the previous advantages and overcoming the difficulties. As it is inspired by the UKF method afterwards it is named Projected Interval Unscented Kalman Filter (PIUKF).

The paper is organized as follows. In the first section, the second order model (Papageorgiou et al., 1990 ) is described. In the second one, the UKF method is presented considering the limitations it presents to be applied to vehicular traffic. In the third section, a variant of the UKF method, called PIUKF, which solves the UKF limitations is presented. In section four, the proposed algorithm is tested using several examples that can be divided in two classes according the simulators used for reference. One based on METANET and the second based on SUMO. Among each class the examples differ in the parameter setting and in the possibility of estimating them.

\section{Traffic Model}

The second order model proposed in (Papageorgiou et al., 1990) is a macroscopic model for traffic simulation. In this model a given freeway stretch is subdivided in $N$ segments of length $l_{i}$ with $\lambda_{i}$ lanes in each segment. The length of the segments must satisfy

$$
l_{i}>T v_{0} \quad \forall i
$$

where $v_{0}$ is the free speed.

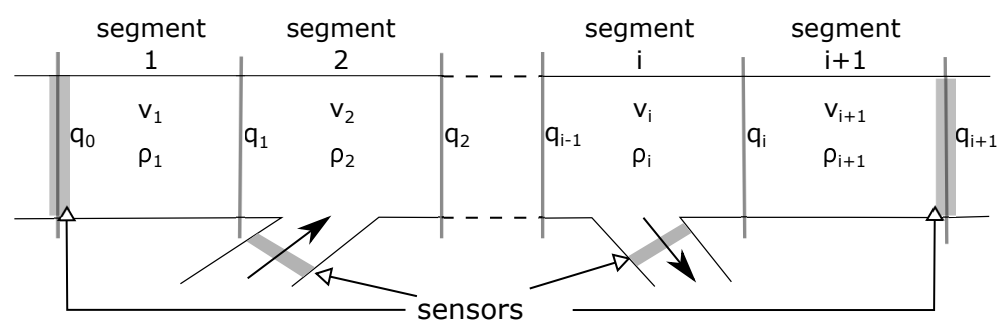

Figure 1: Segment and state variables in a freeway

For each segment the following aggregated traffic variables are defined and shown in the figure 1 :

$q_{k}^{i}$ Traffic flow $(v e h / h)$ is the number of vehicles leaving segment $i$ during the time period $[k T,(k+1) T]$, divided by $T$;

$\rho_{k}^{i}$ Traffic density (veh/km/lane) is the number of vehicles in segment $i$ at time $k T$, divided by the segment length $l_{i}$ and lane number $\lambda_{i}$; 
$v_{k}^{i}$ Mean speed $(\mathrm{m} / \mathrm{h})$ is the mean speed oh the vehicles included in segment $i$ at time $t=k T$;

$r_{k}^{i}, s_{k}^{i}$ On-ramp inflow and Off-ramp outflow $(v e h / h)$ in segment $i$.

These variables verify the following equations. First, the traffic flow is related to the density through :

$$
q_{k}^{i}=\rho_{k}^{i} v_{k}^{i} \lambda_{i}
$$

The evolution of density is given by the following equation

$$
\rho_{k+1}^{i}=\rho_{k}^{i}+\frac{T}{l_{i} \lambda_{i}}\left[q_{k}^{i-1}-q_{k}^{i}+r_{k}^{i}-s_{k}^{i}\right]
$$

which expresses the vehicle conservation principle, and for the speed we have

$$
v_{k+1}^{i}=v_{k}^{i}+\frac{T}{\tau}\left(V\left(\rho_{k}^{i}\right)-v_{k}^{i}\right)+\frac{T}{l_{i}} v_{k}^{i}\left(v_{k}^{i-1}-v_{k}^{i}\right)-\frac{\eta T}{\tau l_{i}} \frac{\left(\rho_{k}^{i+i}-\rho_{k}^{i}\right)}{\rho_{k}^{i}+\kappa}-\frac{\delta T}{\lambda_{i} l_{i}} \frac{r_{k}^{i} v_{k}^{i}}{\rho_{k}^{i}+\kappa},
$$

which is an equation designed to model the dynamic evolution of the mean speed at each segment. The values $\kappa[\mathrm{veh} / \mathrm{km}], \tau[\mathrm{h}], \eta\left[\mathrm{km}^{2} / \mathrm{h}\right]$ and $\delta$ are model parameters which are given the same values for all the segments. Considering the fundamental diagram used in this work (see (Papageorgiou et al., 1990)), the desired speed is related with the segment density as follows

$$
V\left(\rho_{k}^{i}\right)=v_{0} \exp \left(-\frac{1}{a}\left(\frac{\rho_{i}}{\rho_{c}}\right)^{a}\right)
$$

where $\rho_{c}$ is the critical density at which the traffic flow is maximal and $a$ is a model parameter to adjust the shape of the fundamental diagram.

The equations (3-4) model the system dynamics and, representing with $x_{k}$ the vector of all the state variables (density and velocity for each inner segment) at time $k$, they can be written in a compact way as

$$
x_{k+1}=f_{k}\left(x_{k}, u_{k}\right),
$$

where $u_{k}$ is the input that in our case is a vector with the components $q_{0}^{i}, v_{0}^{i}, r_{k}^{i}$ and $s_{k}^{i}$ for all time steps $k$. The measures are grouped in the variable $y_{k}$ and the model equations for the observation is given by

$$
y_{k}=h_{k}\left(x_{k}\right) \text {. }
$$

\section{Estimation}

Basically, the estimation problem consists in solving the large scale system of nonlinear equations defined by the values of $x$ that verify (6-7) given some initial value for $x_{0}$ and the observations $y_{k}$, for $k=1, \ldots, K$. As this inverse problem is not well posed, the standard approach considers that all the equations are affected by Gaussian errors and then rewrites the problem as the minimization of the total error constrained to equations (6-7). The introduction of random errors transforms the deterministic variables (that verify the deterministic difference equations 6-7) into random variables making up a stochastic process. This process must verify the following stochastic difference equations:

$$
\begin{aligned}
x_{k+1} & =f_{k}\left(x_{k}, u_{k}\right)+\xi_{k}, \\
y_{k} & =h_{k}\left(x_{k}, u_{k}\right)+\zeta_{k},
\end{aligned}
$$

where $\xi_{k}$ and $\zeta_{k}$ are the error vectors for the model and the observation, assumed to be normally distributed, with 0 mean and covariance matrices $Q_{k}$ and $R_{k}$. The criterium to obtain the best estimation is to reduce 
the mean squared error and this turns to be equivalent to sum over time the trace of the covariances matrices of $x_{k}-\hat{x}_{k}$ and $y_{k}-\hat{y}_{k}$. When the functions $f$ and $h$ are linear, the classical Kalman filter gives an explicit formula to recursively obtain the solution. When they are nonlinear functions, as in our case, a modified version of the filter called Extended Kalman Filter (EKF) can be applied. An example of that appears in the researchwork (Wang and Papageorgiou, 2005), where the authors present an approach to estimate the state of a freeway applying EKF method and the second order model stated in (Papageorgiou et al., 1990). The EKF linearizes the function $f$ and $h$ around the current estimation $\hat{x}_{k}$, and the equations are, for the Prediction step,

$$
\begin{aligned}
x_{k+1}^{-} & =f\left(\hat{x}_{k}, u_{k}\right), \\
P_{k+1}^{-} & =F_{k} \hat{P}_{k} F_{k}^{T}+Q_{k}
\end{aligned}
$$

and for the Correction step,

$$
\begin{aligned}
& \hat{x}_{k+1}=x_{k+1}^{-}+K_{k+1}\left(z_{k+1}-h\left(x_{k+1}^{-}\right)\right), \\
& \hat{P}_{k+1}=\left(I-K_{k+1} H_{k}\right) P_{k+1}^{-},
\end{aligned}
$$

where $F_{k}$ and $H_{k}$ are the jacobian matrices obtained by linearizing $f$ and $h$ around $\hat{x}_{k}$, and the matrix $K_{k}$ is the Kalman gain computed by

$$
K_{k+1}=P_{k+1}^{-} H_{k}^{T}\left(H_{k} P_{k+1}^{-} H_{k}^{T}+R_{k}\right)^{-1} .
$$

The advantage of the EKF is its recursive formula to compute the estimation, but the validity of the methodology strongly depends on the non linear character of the functions $f$ and $h$. In fact, the update and correction formulae for the covariance matrix $P_{k}$ are exact if the functions are linear and can be considered as good approximations if those functions are aproximatedly linear. This can be explained because of the identity $\operatorname{cov}(A x)=A \operatorname{cov}(x) A^{T}$ but there is no similar identity relating $\operatorname{cov}(x)$ and $\operatorname{cov}(f(x))$ for non linear $f$. When the non linearity affects the performance, a way to circumvect this problem is to estimate the covariance $\operatorname{cov}(f(x))$ instead of using a poorly approximating formula. When this is made using the so called Unscented Tranformation the resulting method is called Unscented Kalman Filter (UKF). As stated in (Valverde and Terzija, 2011), UKF methodology improves the results obtained from EKF in many different areas of application. Following this idea, the aim of this research is to develop a new variant of the UKF method which could be applied to traffic estimation. UKF is a dynamic estimator which combines the Unscented transformation and the Kalman filter to estimate the state of a non-linear system, having that in mind the following topics are introduced.

\section{Unscented Transformation}

The Unscented transformation (Julier and Uhlmann, 2004) allows estimating the distribution of the random variable $y=f(x)$ knowing the function $f$ and the distribution of the random variable $x$. Such transformation consists in choosing a set of points $\left\{X_{i}\right\}$ known as sigma points in such a way that their mean and covariance coincide with the random variable $x$. The function $f$ is then applied to the sigma points and the mean and covariance of $f(x)$ are estimated from $\left\{f\left(X_{i}\right)\right\}$.

More precisely, considering the variable $x$ of dimension $n$ with expectation $E[x]=\hat{x}$ and covariance $\operatorname{cov}(x)=P_{x}$, the $(2 n+1)$ sigma points $X^{0}, X^{1}, \ldots, X^{2 n}$ are defined by

$$
\begin{aligned}
X^{0} & =\hat{x}, \\
X^{j} & =\hat{x}+\gamma^{j}\left(\sqrt{P_{x}}\right)_{j}, \quad j=1, \ldots, n, \\
X^{j} & =\hat{x}-\gamma^{j-n}\left(\sqrt{P_{x}}\right)_{j-n}, \quad j=n+1, \ldots, 2 n,
\end{aligned}
$$


where $\gamma^{i}=\sqrt{(n+\lambda)}$ for $i=1, \ldots, n, \gamma^{0}=0$, and $\lambda=\alpha^{2}(n+\nu)-n$. We let $\gamma^{i}$ depend on the index $i$ even if it is constant because in the next subsections we will change its definition. Then, the expectation and covariance is calculated as

$$
\begin{gathered}
\bar{x}=\sum_{j=0}^{2 n} W_{j}^{(m)} X^{j}, \\
P_{x}=\sum_{j=0}^{2 n} W_{j}^{(c)}\left[X^{j}-\bar{x}\right]\left[X^{j}-\bar{x}\right]^{T},
\end{gathered}
$$

where the weight values are defined as follows

$$
\begin{aligned}
W_{0}^{(m)} & =\frac{\lambda}{n+\lambda}, \\
W_{0}^{(c)} & =\frac{\lambda}{n+\lambda}+1-\alpha^{2}+\beta, \\
W_{j}^{(m)} & =W_{j}^{(c)}=\frac{1}{2(n+\lambda)}, j=1, \ldots, 2 n .
\end{aligned}
$$

The parameter $\alpha$ determines the spread of the sigma points around $\bar{x}$ and usually takes a value inside the interval $\left(10^{-4}, 1\right)$. The parameter $\beta$ is used to incorporate the knowledge of the distribution of $\bar{x}$ (for Gaussian distributions $\beta=2$ ), $\nu$ is usually 0 . All of the parameters were obtained from (Van der Merwe and Wan, 2001).

To estimate the statistics of $f(x)$, the equations (18) and (19) are used with the same weights but changing the sigma points with their images through $f$, i.e., changing $X^{j}$ for $Y^{j}=f\left(X^{j}\right)$ :

$$
\begin{aligned}
\bar{y} & \approx \sum_{j=0}^{2 n} W_{j}^{(m)} Y^{j} \\
P^{y} & \approx \sum_{j=0}^{2 n} W_{j}^{(c)}\left[Y^{j}-\bar{y}\right]\left[Y^{j}-\bar{y}\right]^{T} .
\end{aligned}
$$

\section{Unscented Kalman Filter}

As it was said before, the Unscented Kalman Filter is a combination of the the classical Kalman filter (1114) with the unscented transformation (15-24). It was firstly presented in the work (Julier and Uhlmann, 2004) and after coputing the sigma points with (15-17) it makes the following two steps:

Prediction

$$
\begin{gathered}
X_{k+1}^{j-}=f_{k}\left(X_{k}^{j}, u_{k}\right), \\
x_{k}^{-}=\sum_{j=0}^{2 n} W_{j}^{(m)} X_{k+1}^{j-}, \\
P_{k}^{-}=\sum_{j=0}^{2 n} W_{j}^{(c)}\left[X_{k+1}^{j-}-x_{k}^{-}\right]\left[X_{k+1}^{j-}-x_{k}^{-}\right]^{T}+Q_{k}, \\
X_{k+1}^{j-}=\left\{\begin{array}{l}
x_{k}^{-}+\gamma^{j}\left(\sqrt{P_{k}^{-}}\right)_{j}, j=0, \ldots, n, \\
x_{k}^{-}-\gamma^{j-n}\left(\sqrt{P_{k}^{-}}\right)_{j-n}, j=n+1, \ldots, 2 n,
\end{array}\right.
\end{gathered}
$$




$$
\begin{gathered}
Y_{k}^{j}=h_{k}\left(X_{k+1}^{j-}, u_{k}\right), \\
y_{k}^{-}=\sum_{j=0}^{2 n} W_{j}^{(m)} Y_{k}^{j} .
\end{gathered}
$$

\section{Correction}

$$
\begin{gathered}
P_{y_{k}^{-} y_{k}^{-}}=\sum_{j=0}^{2 n} W_{j}^{(c)}\left[Y_{k}^{j}-y_{k}^{-}\right]\left[Y_{k}^{j}-y_{k}^{-}\right]^{T}+R_{k}, \\
P_{x_{k}^{-} y_{k}^{-}}=\sum_{j=0}^{2 n} W_{j}^{(c)}\left[X_{k+1}^{j-}-x_{k}^{-}\right]\left[Y_{k}^{j}-y_{k}^{-}\right]^{T}, \\
K_{k}=P_{x_{k}^{-} y_{k}^{-}} P_{y_{k}^{-} y_{k}^{-}}^{-1} \\
\hat{x}_{k+1}=x_{k}^{-}+K_{k}\left(y_{k}-y_{k}^{-}\right), \\
P_{k+1}=P_{k}^{-}-K_{k} P_{y_{k} y_{k}} K_{k}^{T} .
\end{gathered}
$$

The sigma points are accommodated in the matrix $X_{k}$ and corresponds to the current estimation plus and minus $\sqrt{P}_{k}$, the square root matrix of the covariance. This matrix is analogous to the one dimensional standard deviation and it is well defined because $P_{k}$ is positive semidefinite. During numerical computation, in order to avoid numerical errors that could be incurred in the computation of $\sqrt{P}$, the Cholesky decomposition of $P$ is used and each factor is updated iteratively (Julier et al., 2000).

Using the model represented in the section above, two state variables for each segment of the freeway are considered: density $\rho$ and average speed $v$. When the sigma points are computed considering equation (38), it is possible to obtain non realistic values of $\rho$ and $v$ that fall outside the model domain, for instance, a negative speed. To depict such situation consider the following example: there is only one segment with density $\rho=0.8 \mathrm{veh} / \mathrm{km} /$ lane, average speed $v=0.3 \mathrm{~km} / \mathrm{h}$ and variances $\sigma_{\rho}^{2}=0.5, \sigma_{v}^{2}=0.5$ respectively. The resulting sigma points are shown in figure 2 , where it can be observed that the sigma point $X_{3}$ gets a negative speed value and $X_{4}$ a negative density, what is not actually possible. What is worse, the dynamic equations of the model (3-5) can not be applied to these values.

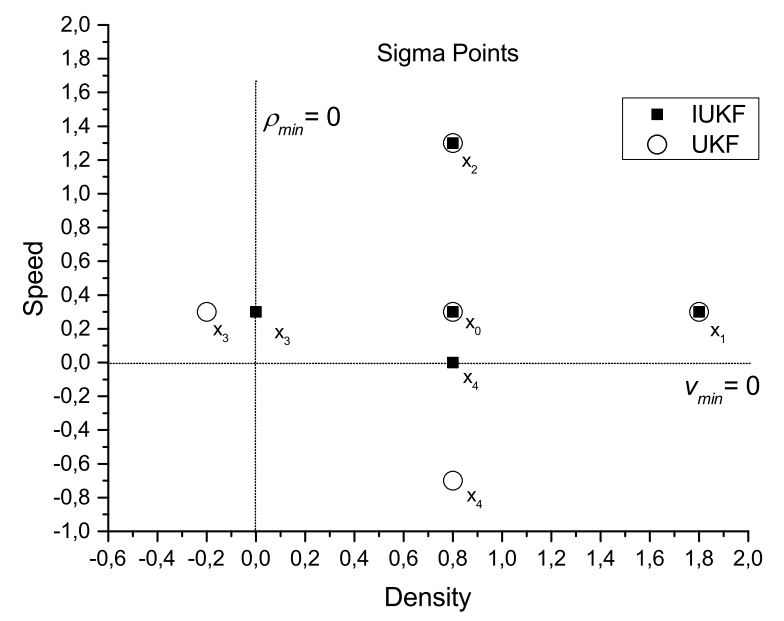

Figure 2: Sigma Points obtained using UKF and IUKF 


\section{Constrained versions of the UKF method}

In (Teixeira et al., 2008) a method called Interval Unscented Kalman Filter (IUKF) is presented. This method is a variant of the Unscented Kalman Filter that adds constraints to the sigma points fixing the problem of computation of the dynamic equations outside their validity domain.

The IUKF methods assumes that the following box constraints should be satisfied by the state variables $x_{k}$ :

$$
d_{k} \leq x_{k} \leq e_{k}
$$

where $d_{k}$ and $e_{k}$ are lower and upper bounds of $x_{k}$. In order to have all the sigma points inside the feasible domain, it redefines the parameters $\gamma$ and the weights $W$ in equations (15-22) that will now depend on time step $k$. They introduce the matrix $S_{k}=\left[\sqrt{P_{k}}-\sqrt{P_{k}}\right]$ and the matrix $\Gamma$ given by

$$
\Gamma_{k}^{i j}=\sqrt{n+\lambda}-\left.\left(\sqrt{n+\lambda}-\frac{e_{k}^{i}-x_{k}^{i}}{S_{k}^{i j}}\right)^{+}\right|_{S_{k}^{i j}>0}-\left.\left(\sqrt{n+\lambda}-\frac{d_{k}^{i}-x_{k}^{i}}{S_{k}^{i j}}\right)^{+}\right|_{S_{k}^{i j}<0},
$$

where the index $i=1, \ldots, n$ corresponds to the variable space and the index $j=1, \ldots, 2 n$, to the sigma points. Finally defining $\gamma_{k}^{i}=\min _{j} \Gamma_{k}^{i j}$ they obtain the feasible sigma points

$$
X_{k}=\left[\begin{array}{lll}
\hat{x}_{k} & \hat{x}_{k}+\gamma_{k}^{i}\left(\sqrt{P_{k}}\right)_{i} & \hat{x}_{k}-\gamma_{k}^{i}\left(\sqrt{P_{k}}\right)_{i}
\end{array}\right],
$$

and the corresponding weights are

$$
W_{0, k}^{(m)}=W_{0, k}^{(c)} \triangleq b_{k}, \quad W_{j, k}^{(m)}=W_{j, k}^{(c)} \triangleq a_{k} \gamma_{k}^{j}+b_{k},
$$

where

$$
\tau_{k}=\sum_{j=1}^{n} \gamma_{k}^{j}-(2 n+1) \sqrt{n+\lambda} ; \quad a_{k}=\frac{2 \lambda-1}{2(n+\lambda) \tau_{k}} ; \quad b_{k}=\frac{\tau_{k}-2 \lambda+1}{2(n+\lambda) \tau_{k}} .
$$

Considering the example shown in figure 2, but computing the sigma points using the IUKF method with bounds $\rho_{\min }=0 \mathrm{veh} / \mathrm{km} /$ lane, $\rho_{\max }=100 \mathrm{veh} / \mathrm{km} / \mathrm{lane}, v_{\min }=0 \mathrm{~km} / \mathrm{h}$ y $v_{\operatorname{mix}}=150 \mathrm{~km} / \mathrm{h}$, the corresponding sigma point are shown in figure 2 .

As it is observed, the density value of the sigma point $X_{3}$ is proyected to 0 and the speed value of the sigma point $X_{4}$ is proyected to 0 , considering the lower bound limits.

Even though IUKF fixes the problems with the dynamic equations, the correction step (34) can give an estimation outside the validity domain of the model and in the next iteration the sigma points could not be computed.

A method that after each iteration finishes with a valid point is also presented in (Teixeira et al., 2008) and called Projected Unscented Kalman filter (PUKF). This method applies a projection to the UKF result in order to force the estimated value to be in the chosen domain. This approach applies the prediction and the correction step of the UKF method and then gets the estimation $\hat{x}_{k+1}$ in step (34) solving the following optimization problem:

$$
\hat{x}_{k+1}^{P}=\arg \min _{d_{k} \leq x_{k} \leq e_{k}} J_{2}\left(x_{k}\right),
$$

where

$$
J_{2}\left(x_{k}\right)=\left(x_{k}-\hat{x}_{k+1}\right)^{T} W_{k}^{-1}\left(x_{k}-\hat{x}_{k+1}\right)
$$

and $W_{k}^{-1}$ is a weight matrix associated with the state variable. The resolution of the optimization problem (41) is made to project the estimated value to the feasible set.

As it is, this method cannot be applied to our problem because it has the same difficulties for the sigma points shown in the previous section. The IUKF does not have these problems but the correction 
step (41) can give an estimation outside this domain. With this in mind, a combination of both methods is proposed as a new method called Projected Interval Unscented Kalman Filter (PIUKF). Precisely, this new method, consists in adding the projection step (42) after the correction step of IUKF. The IUKF part gives valid sigma points and the PUKF part restores the corrected estimation to the validity domain. Nevertheless, it should be mentioned that no projection is applied to the covariance matrix $P_{k+1}$. To resume, the proposed PIUKF method is defined by:

Prediction equations (25-30) with sigma points given by (??-??) and weights given by (39-??),

Correction equations (31-35) with weights given by (39-??),

Projection equations (41-42).

\section{Results}

The examples can be divided into two classes according to the simulation tool involved METANET and SUMO

METANET (Messner and Papageorgiou, 1990) is a software for freeway traffic simulation based on a macroscopic second order model. The overall modelling approach allows for simulation of several traffic conditions and incidents. It may be applied to multi-origin, multi-destination, multi-route freeway networks with arbitrary topology including bifurcations, junctions, on-ramps and off-ramps. Simulation results are provided in terms of macroscopic traffic variables such as traffic density, traffic volume, and mean speed at all network segments as well as in terms of travel times on selected routes.

SUMO (Krajzewicz et al., 2002) is an open source traffic simulation package which also has tools to import network topologies and to model demand parameters. It is a purely microscopic traffic simulation. A route through the whole network is explicitely assigned to each vehicle together with its characteristics that describe the vehicle's physical properties and the variables of the used movement model. The simulation approach is time-discrete and space-continuous. Internally each vehicle position is described by the lane the vehicle is on and the distance from the beginning of this lane. When moving through the network, each vehicle speed is computed using the car-following model which is an extension of the one developed by Stefan Krauß(Krauß, 1998). Simulation results include induction loop observations and single vehicle positions at each time steps for all vehicles or aggregated measures along a street or lane.

It is important to remark that the simulation methodology of METANET is based on the same equations used to derive the estimation algorithm. On the other hand, SUMO is a microscopic simulation software and hence the output could be considered more likely as real observed data.

In order to assess the performance of the method, the following indexes were taken from the work (Wang and Papageorgiou, 2005). They represent for each time step, the mean square error for the estimated density and speed:

$$
I_{k}^{\rho}=\sqrt{\frac{1}{N} \sum_{i=1}^{N}\left[\rho_{k}^{i}-\rho_{k}^{i *}\right]^{2}}, \quad I_{k}^{\rho}=\sqrt{\frac{1}{N} \sum_{i=1}^{N}\left[v_{k}^{i}-v_{k}^{i *}\right]^{2}} .
$$

where $v_{i}^{*}$ and $\rho_{i}^{*}$ are the estimated speed and density in segment $i$.

To compare the overall performance of the methods, we will also consider averaged-on- time versions of the indexes

$$
I^{\rho}=\sqrt{\frac{1}{K N} \sum_{k=0}^{K} \sum_{i=1}^{N}\left[\rho_{k}^{i}-\rho_{i}^{*}(k)\right]^{2}}, \quad I^{v}=\sqrt{\frac{1}{K N} \sum_{k=0}^{K} \sum_{i=1}^{N}\left[v_{k}^{i}-v_{i}^{*}(k)\right]^{2}},
$$

where $K$ is the number of simulated steps. 
In the first case, the EKF and the PIUKF methods are applied considering two different perturbation schemes. Numerical results are obtained using the METANET (Messner and Papageorgiou, 1990) simulator applied to a scenario presented in (Wang and Papageorgiou, 2005).

In the second case, a similar scenario is considered performing the numerical simulation with SUMO (Behrisch et al., 2011). It should be mentioned that this simulator is based in a car-following approach, which considers a more detailed model than the one used in the estimation algorithm.

Finally, both approaches were compared considering the performance indexes proposed in (Wang and Papageorgiou, 2005).

\section{Test case 1: METANET Simulation}

In this case the measures observed in the freeway presented in figure 3 are simulated using METANET (Messner and Papageorgiou, 1990). The freeway stretch is divided into 12 segments, (numbered from 1 to 12) each with a length of $500 \mathrm{~m}$. Segments $1-11$ have three lanes with a maximum flow of $2000 \mathrm{veh} / \mathrm{h}$ each one while segment 12 has only two lanes with a maximum flow of $1500 \mathrm{veh} / \mathrm{h}$ each one. The stretch also has one on-ramp and one off-ramp in segment 7 and 9 respectively.

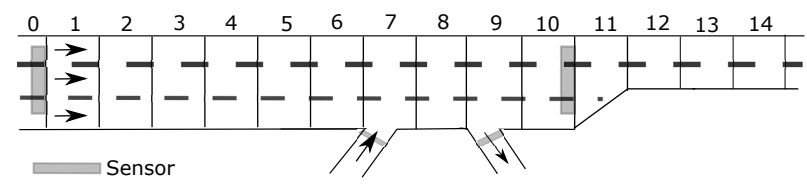

Figure 3: Traffic network simulated with METANET

As observed in figure 3, two detectors are installed respectively at the upper boundary of segment 1 and lower boundary of segment 10, and two more detectors are installed at the on-ramp and at the off-ramp. These detectors measures the mean speed $v$ and the traffic flow $q$. The simulation period lasts 6 hours from 7:00 to 13:00 and a special traffic scenario is designed so as to produce several traffic conditions. More specifically, the incoming-flow of segment 12 exceeds its maximum permissible flow (3000 veh/h/lane) during the peak period (10:30:11:00), and a traffic incident occurs in segment 12 at around 7:33 and lasts 33 min, during which one lane in segment 12 is blocked. The congestion propagates upstream into the estimated freeway stretch as seen in the figure (5). Simulation parameters are $v_{0}=120 \mathrm{~km} / \mathrm{h}, \rho_{c}=33.5 \mathrm{veh} / \mathrm{km} /$ lane and $a=1.5324$, for each segment. The minimum and maximum density are $\rho_{\min }=0 \mathrm{veh} / \mathrm{km} /$ lane and $\rho_{\max }=100 \mathrm{veh} / \mathrm{km} /$ lane respectively, while the speed constraints are $v_{\min }=0 \mathrm{~km} / \mathrm{h}$ and $v_{\max }=120 \mathrm{~km} / \mathrm{h}$ for each section. The standard deviations for the model errors are $0.04 \mathrm{veh} / \mathrm{km} /$ lane for the density and $10 \mathrm{~km} / \mathrm{h}$ for the speed, and for the observation errors are $100 \mathrm{veh} / \mathrm{h} /$ lane for the flow and $20 \mathrm{~km} / \mathrm{h}$ for the speed.

In the work (Wang and Papageorgiou, 2005), the EKF is studied for four different schemes, that differs in the definition of state variables, inputs and outputs. There, they show that no advantage is obtained with schemes more involved than the first one which was the simpler one for calculations. Therefore, this paper takes the first of those schemes that consists in considering all measures as observations and no input at all. The same observation applies to the different detector configurations studied in that paper. Here we retain the first one consisting in 4 detectors conveniently placed. More precisely, the average speed $v_{10}$ and flow $q_{10}$ of the detector located between segments 10 and 11 are considered as measures. The measures of the detectors 1, 3 and 4 are used as inputs to the method. The parameters of the fundamental diagram (5) are considered known (hence not estimated) and the value of the exponent is $a=1.5324$.

The figures 4 and 5 show how the state variables behave in the freeway segments 6 and 11, where different perturbations are presented. In figure 4 density and speed results for the segment 6 (located before the entrance ramp) are presented. 

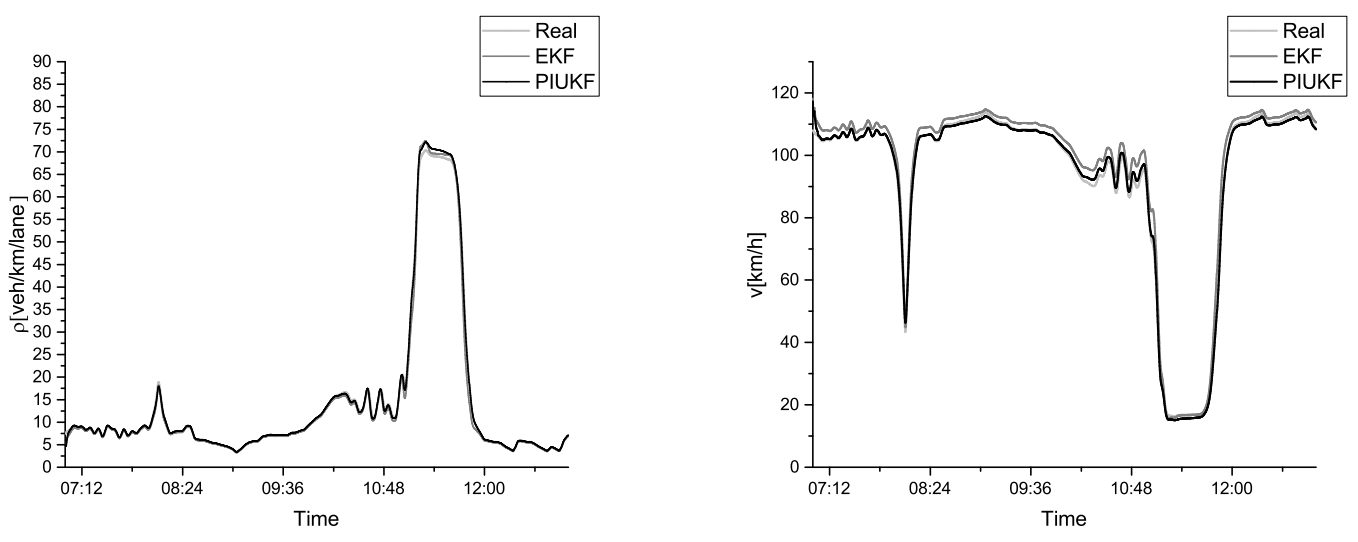

Figure 4: Segment 6 Density (left) and Speed (right)

The figure 4 shows how the incident occurred upstream (from 10:30 to 11) affects both density and speed. Although both methods accurately estimate real values, a more detailed analysis (in the right diagram) shows that the PIUKF method is closer to the real speed values than the EKF. The density values estimated by both approaches are very close to the real ones (left diagram). In figure 5, the same analysis is done for the segment 11 .
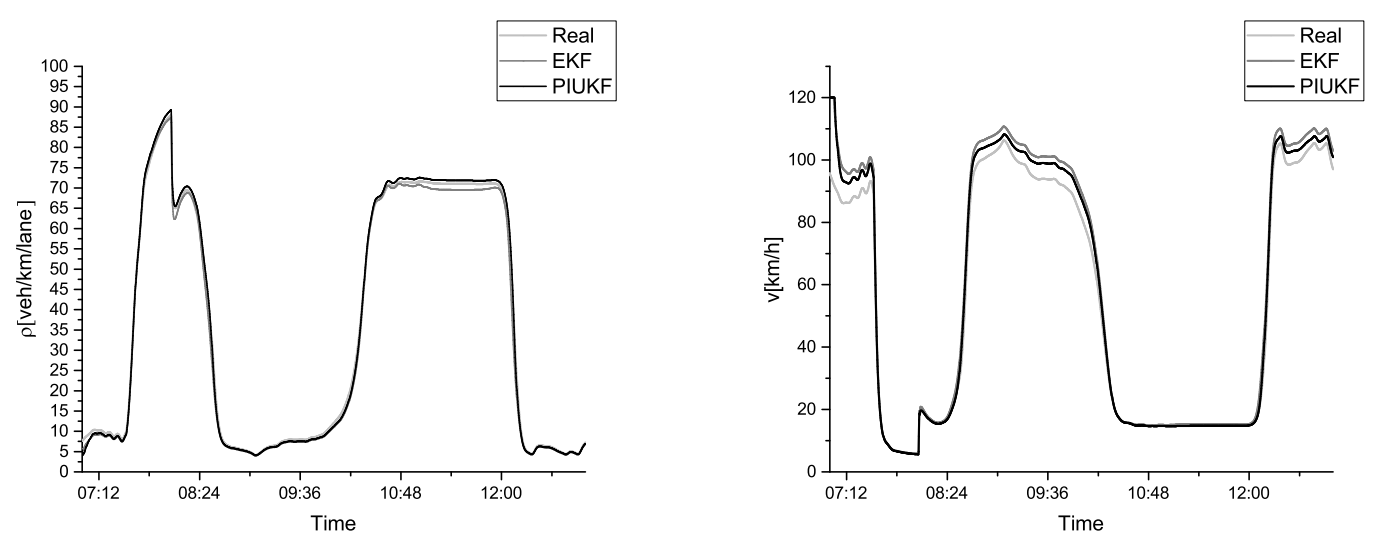

Figure 5: Segment 11 Density (left) and Speed (right)

As shown in the figure 5, the congestion caused by the incident lasts longer in the segment 11 than in the segment 6. It is observed that the EKF and PIUKF methods accurately estimate the real values. A similar performance to the previous segments estimation is observed in the other segments of the network, the results for each segment are summarized in the table 1.

In the figure 6 the error with respect to the simulated reference case is presented. It can be seen that the PIUKF method performs better specially in speed estimation. Considering the density variable, the PIUKF recovers faster from a disturbance (from 10:30 to 11:00 and from 7:33 to 8:06). 


\begin{tabular}{c|ccc|ccc|}
\multirow{2}{*}{ Segment } & \multicolumn{4}{|c|}{$P I_{\rho}$} & \multicolumn{4}{c|}{$P I_{v}$} \\
& EKF & PIUKF & Improvement & EKF & PIUKF & Improvement \\
\hline & & & & & & \\
1 & 1.17 & 1.06 & $9.01 \%$ & 9.48 & 9.02 & $4.84 \%$ \\
2 & 1.12 & 0.87 & $22.44 \%$ & 7.07 & 5.56 & $21.39 \%$ \\
3 & 1.58 & 1.03 & $35.04 \%$ & 5.16 & 3.06 & $40.75 \%$ \\
4 & 1.32 & 0.65 & $50.91 \%$ & 4.03 & 1.75 & $56.57 \%$ \\
5 & 1.19 & 0.55 & $53.75 \%$ & 3.69 & 1.23 & $66.6 \%$ \\
6 & 1.24 & 0.54 & $56.44 \%$ & 3.72 & 1.12 & $69.84 \%$ \\
7 & 1.35 & 0.57 & $57.99 \%$ & 3.77 & 1.18 & $68.58 \%$ \\
8 & 1.51 & 0.72 & $52.71 \%$ & 3.8 & 1.25 & $67.25 \%$ \\
9 & 1.44 & 0.78 & $45.62 \%$ & 3.2 & 1.17 & $63.45 \%$ \\
10 & 1.39 & 0.9 & $35.16 \%$ & 3.29 & 1.39 & $57.73 \%$ \\
11 & 1.47 & 0.97 & $33.67 \%$ & 5.92 & 4.51 & $23.84 \%$
\end{tabular}

Table 1: The indexes $P I_{v}$ and $P I_{\rho}$ for all the segments simulated with METANET and the improvement percentage
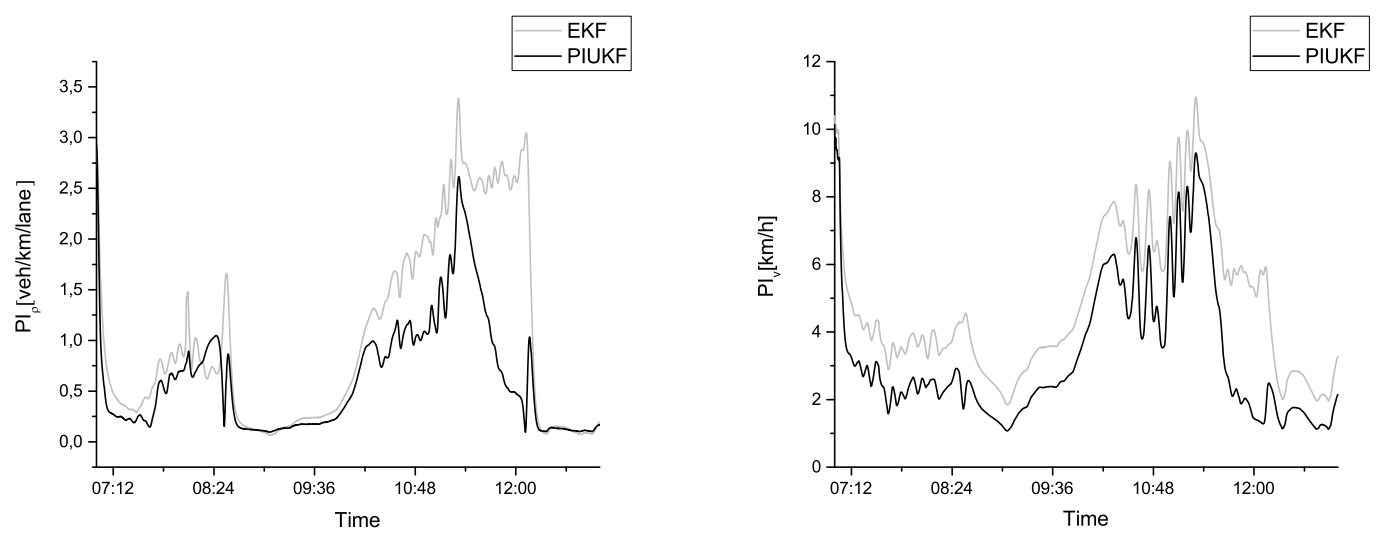

Figure 6: $I_{k}^{\rho}$ (left) and $I_{k}^{v}$ (right) obtained for EKF and PIUKF methods simulating with METANET

\section{Test Case 2: SUMO Simulation}

A second test case is performed using a microscopic simulator called SUMO (Behrisch et al., 2011) and considering both previously mentioned estimation methods. A similar network to the first case is created using SUMO but defining a higher inflow in order to produce congestion in higher segments. The considered freeway is presented in Figure 7 and has 14 segments. Segments 1 to 11 are under study and have two lanes, while segment 12 to 14 have a single lane. The stretch also has one on-ramp and one off-ramp in segment 7 and 9 respectively. The minimum and maximum density are $\rho_{\min }=0$ veh $/ \mathrm{km} /$ lane and $\rho_{\max }=$ $100 \mathrm{veh} / \mathrm{km} / \mathrm{lane}$ respectively, while the speed constraints are $v_{\min }=0 \mathrm{~km} / \mathrm{h}$ and $v_{\max }=130 \mathrm{~km} / \mathrm{h}$ for each segment. The standard deviations for the model errors are $0.08 \mathrm{veh} / \mathrm{km} /$ lane for the density and $9 \mathrm{~km} / \mathrm{h}$ for the speed, and for the observation errors are $90 \mathrm{veh} / \mathrm{h} /$ lane for the density and $8 \mathrm{~km} / \mathrm{h}$ for the speed. Car-following parameters are the default values used by the SUMO simulator. It should be mentioned that the car-following model considered in this test case simulates a more detailed vehicle interaction dynamic that the one considered in the previous test case. 


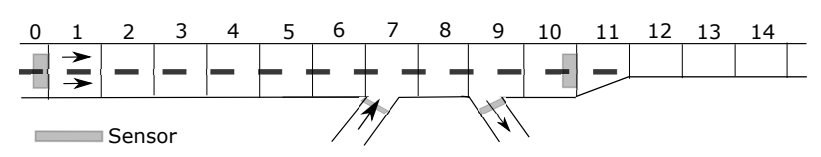

Figure 7: Network simulated using SUMO

Figures 8 and 9 shows density and speed obtained for segments 3 and 6 . In these figures is noted that when the most important non-linearities occur (periods when congestion begins) the PIUKF method values are closer to real results. This fact is possible because the PIUKF method is based on the UKF method, which has a better approximation of the error covariance matrix when more important non-linearities occur.
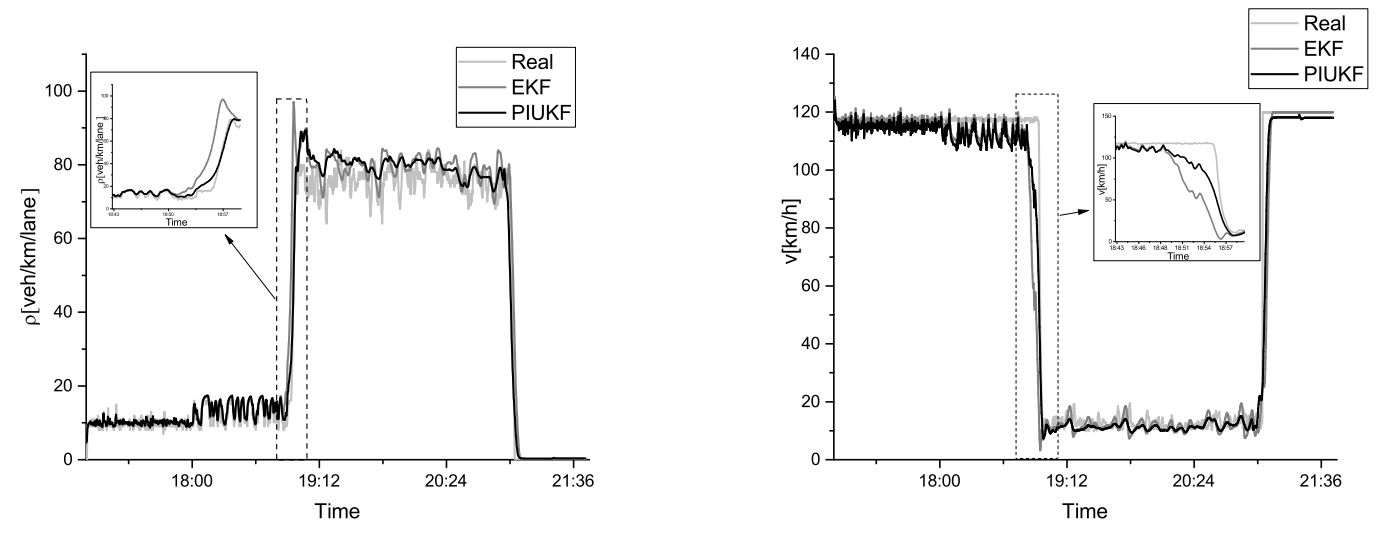

Figure 8: Segment 3 Density (left) and Speed (right)
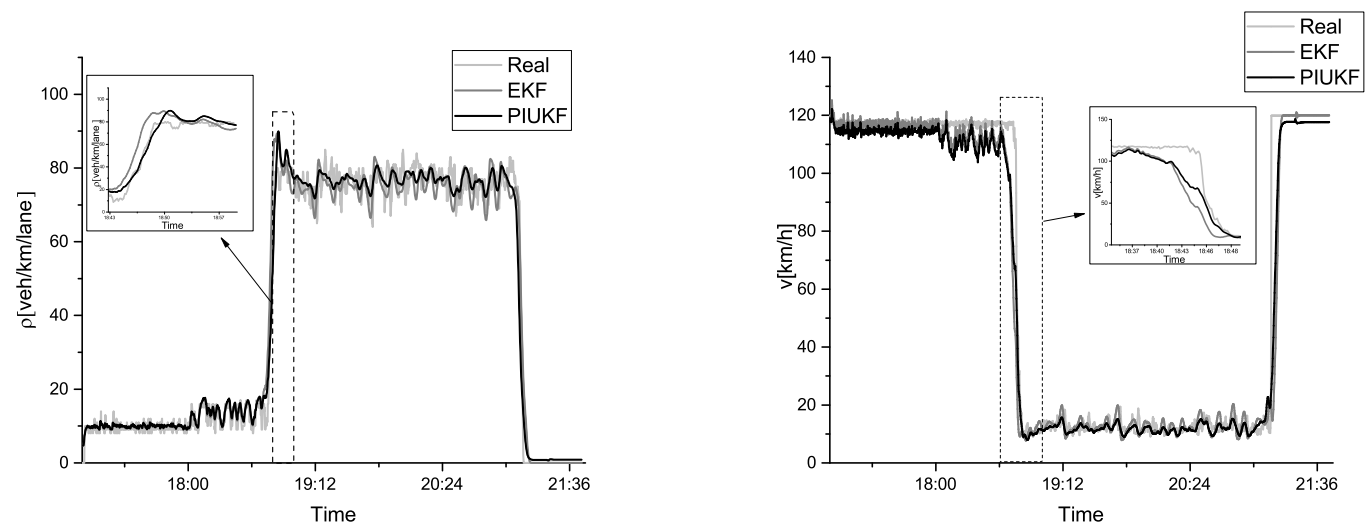

Figure 9: Segment 6 Density (left) and Speed (right)

In the figure 10 the same indexes (43) are shown for the SUMO test case. Here, it is also observed that PIUKF has a lower error than EKF.

\section{Result Analysis}

In both figures 6 and 10 and for both variables, density and speed, the PIUKF method outperforms the EKF. In the table 1 , it is shown that the improvement is obtained in all of the segments. 

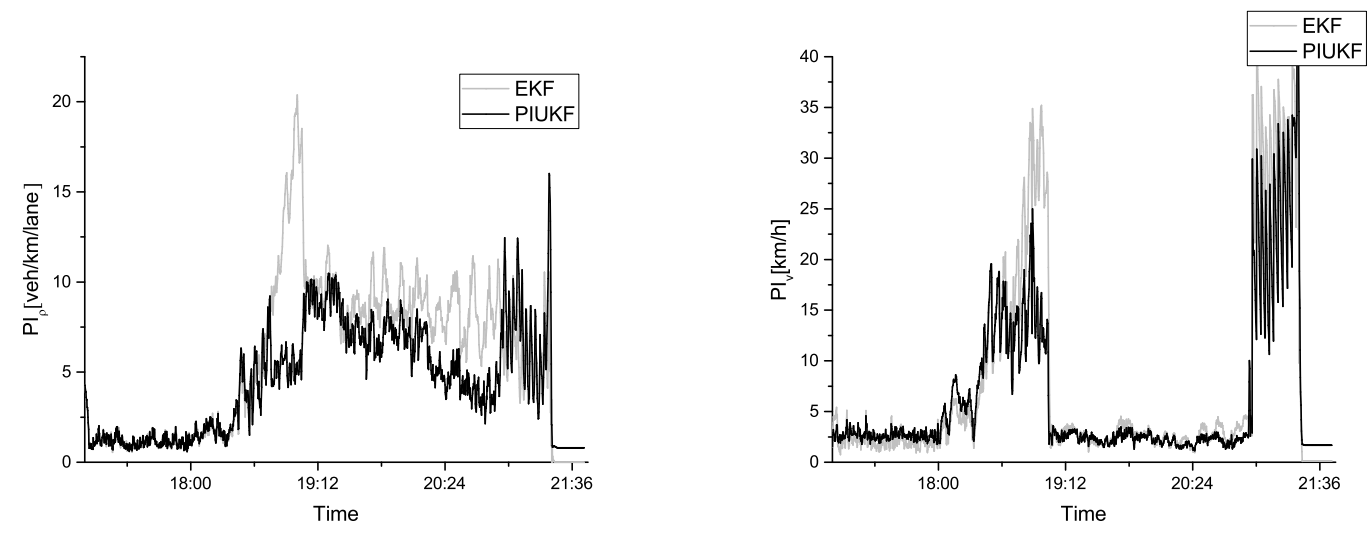

Figure 10: $I_{k}^{\rho}$ (left) and $I_{k}^{v}$ (right) obtained for EKF and PIUKF methods simulating with SUMO

We have also made other simulations over variations of the fore mentioned examples. These variations has to do with the values of the parameters of the Fundamental Diagram (FD) see (5). In the case of METANET we consider the case of a wrong value of the parameter $a$ and the possibility of estimating FD parameters. Thus, we have two more examples in the METANET class:

M1 the example defined in the previous section,

M2 the same case as M1 but during the estimation the FD parameters are estimated starting with a wrong value of $a=1.8$.

Regarding the SUMO class, we have defined 6 more examples.

S1 is the previous mentioned example, where the FD parameters were estimated off-line through many simulations.

S2 It is the case $\mathrm{S} 1$, with a wrong value of $a=2.1$.

S3 It is the case S2, starting with a wrong value of $a_{0}=2.1$ and estimating FD parameters.

S4 It is the case S1, with a wrong value of $a=1.8$.

S5 It is the case S4, starting with a wrong value of $a_{0}=1.8$ and estimating FD parameters.

S6 It is the case S1, with a wrong value of $a=1.8$ and $\rho_{c}=37 \mathrm{veh} / \mathrm{km} /$ lane.

S7 It is the case S6, starting with a wrong value of $a_{0}=1.8$ and $\rho_{c}=37$ veh $/ \mathrm{km} / \mathrm{lane}$. and estimating FD parameters.

The values of the errors in all these cases are shown in Table 2. 


\begin{tabular}{l|cc|cc|cc|}
\multirow{2}{*}{ Test Case } & \multicolumn{2}{|c|}{ EKF } & \multicolumn{2}{c|}{ PIUKF } & \multicolumn{2}{c|}{ Improvement over EKF } \\
& $I^{v}$ & $I^{\rho}$ & $I^{v}$ & $I^{\rho}$ & $I^{v}$ & $I^{\rho}$ \\
M1 METANET refence case & 5.18 & 1.35 & 3.74 & 0.81 & $27.8 \%$ & $40 \%$ \\
M2 estimated valued of $a$ & 8.16 & 3.06 & 5.35 & 2.33 & $34.4 \%$ & $23.8 \%$ \\
S1 SUMO Reference case & 12.66 & 7.15 & 9.85 & 5.35 & $22.2 \%$ & $25.2 \%$ \\
S2 wrong $a=2.1$ & 12.73 & 7.13 & 9.78 & 5.46 & $23.2 \%$ & $23.4 \%$ \\
S3 FD parameter estimation & 15.26 & 8.96 & 9.77 & 6.6 & $36.2 \%$ & $26.3 \%$ \\
S4 wrong $a=1.8$ & 14.07 & 7.97 & 10.03 & 6.44 & $28.7 \%$ & $19.2 \%$ \\
S5 FD parameter estimation $a$ & 16.33 & 8.82 & 9.79 & 6.61 & $40.0 \%$ & $25.0 \%$ \\
S6 wrong $a=1.8$ and $\rho_{c}=37$ & 13.93 & 8.55 & 10.05 & 7.95 & $27.8 \%$ & $7.0 \%$ \\
S7 FD parameter estimation & 17.85 & 11.07 & 9.78 & 6.61 & $45.2 \%$ & $40.3 \%$
\end{tabular}

Table 2: $I^{v}$ and $I^{\rho}$ for the simulated cases with METANET and SUMO

As can be seen in both performed estimations, (one using METANET and the other using SUMO), the PIUKF method behaves better than the EKF method estimating both, speed and density. The improvement is more important in the cases where the FD parameters are estimated (and in the real implementation this should be the case) obtaining improvements ranging from $25 \%$ to $40 \%$ for density and from $36 \%$ to $45 \%$ for velocity. In the values simulated using SUMO we believe that this difference is greater because of the unscented transformation that should behave better in presence of nonlinearities.

Also in the table 2, it can be seen that the overall errors of METANET are smaller that SUMO, this can be justified by the fact that in the case of METANET, the same dynamic model is used for estimation and simulation. Instead, SUMO uses a completely different dynamic model based on car-following equations and this surely increases the modeling error that appears in all the analyzed Kalman variants. Ideally, it would have been better that observed data come from real sensors, in this case they were simulated with SUMO which reflects real situations in a fairly accurate way.

\section{Conclusion}

In the area of traffic, the state estimation problem has been considered in this work and solved using methods that can be seen as variants of the classical Kalman filter.

The Extended Kalman filter and the Unscented Kalman filter were reported to be useful tools for the estimation of non linear problems and for many practical applications with nonlinear dynamics, the unscented version outperforms the extended one. In the case of traffic the Extended Kalman filter also proved to be useful in the literature, and with regards to the UKF method, only a few works have applied it but to a first order model.

In this work we showed that a constrained version of the Unscented Kalman filter applied to a second order model, further improves the numerical results. This better results are explained due to the restriction of the estimated values to be inside the natural domain of the variables.

The numerical simulations were made with a macroscopic simulator named METANET and a microscopic one called SUMO. The simulated trajectories are as good with the constrained version as they are with the other methods. Nevertheless, a performance index that is used to compare the estimation with the real state, gives better results with the proposed methodology. More precisely, for the reported cases, the improvement in the performance index is as much as $28 \%$ in speed and $40 \%$ in density for the METANET simulation, and $45 \%$ in speed and $40 \%$ in density for SUMO. It was also shown that the estimation of hidden parameters can be done better with the UKF, and this also has to do with the non linearity of the equations where those parameters appear.

Other investigations are being done in real situations using other kinds of observations like video 
streaming, probe vehicles and wireless sensors. This will be the subject of a future publication.

\section{Acknowledgement}

We would like to show our gratitude to Professor M. Papageorgiou and to Dr. Albert Messmer for providing a time-limited version of the METANET software. With this tool several examples were numerically simulated which allowed a comparison with other approaches. This work was partially supported by CONICET (PIP 2012-2014 Nro. 0286) and FONCyT (Pict 2012-2212) Argentina.

\section{REFERENCES}

Stanciu, E. A., I. M. Moise, and L. M. Nemtoi, Optimization of urban road traffic in Intelligent Transport Systems. In Applied and Theoretical Electricity (ICATE), 2012 International Conference on, IEEE, 2012, pp. 1-4.

Papageorgiou, M. and J.-M. Blosseville, Macroscopic modeling of traffic flow on the Boulevard Peripherique in Paris. Transportation Research B 24, , No. 23B, 1990, pp. 29-47.

Papageorgiou, M., J.-M. Blosseville, and H. Haj-Salem, Modeling and real-time control of traffic flow on the southern part of Boulevard Peripherique in Paris. Part I: modeling, Transportation Research A 24, 1990.

Seo, T., A. M. Bayen, T. Kusakabe, and Y. Asakura, Traffic state estimation on highway: A comprehensive survey. Annual Reviews in Control, Vol. 43, 2017, pp. 128-151.

Simon, D., Kalman filtering with state constraints: a survey of linear and nonlinear algorithms. IET Control Theory Applications, Vol. 4, No. 8, 2010, pp. 1303-1318.

Szeto, M. W. and D. C. Gazis, Application of Kalman Filtering to the Surveillance and Control of Traffic Systems. Transportation Science, Vol. 6, No. 4, 1972, pp. 419-439.

Treiber, M. and D. Helbing, Reconstructing the spatio-temporal traffic dynamics from stationary detector data. Cooperative Transportation Dynamics, 2002.

Hoogendoorn, S. P. and P. H. Bovy, State-of-the-art of vehicular traffic flow modelling. Proceedings of the Institution of Mechanical Engineers, Part I: Journal of Systems and Control Engineering, Vol. 215, No. 4, 2001, pp. 283-303.

Bhouri, N., H. Hadj-Salem, M. Papageorgiou, and J.-M. Blosseville, Estimation of Traffic Density on Motorways Using Presence Detector Measurements. IFAC Proceedings Volumes, Vol. 22, No. 6, 1989, pp. 453 - 457, iFAC Symposium on Advanced Information Processing in Automatic Control (AIPAC'89), Nancy, France, 3-5 July 1989.

Daganzo, C. F., The cell transmission model: a dynamic representation of highway traffic consistent with the hydrodynamic theory. Transportation Research Part B, Vol. 28, No. 4, 1994.

Daganzo, C. F., The cell transmission model, part II: Network traffic. Transportation Research Part B: Methodological, Vol. 29, No. 2, 1995, pp. 79 - 93.

Mihaylova, L. and R. Boel, A Particle Filter for Freeway Traffic Estimation. Decision and Control, 2004. CDC. 43rd IEEE Conference, 2004. 
Mihaylova, L., R. Boel, and A. HEGYI, An unscented Kalman filter for freeway traffic estimation. In Proceedings of 11th IFAC Symposium on Control in Transportation Systems (H. Van Zuylen and F. Middelham, eds.), 2006, pp. 31-36.

Mihaylova, L., R. Boel, and A. Hegyi, Freeway traffic estimation within particle filtering framework. Automatica, Vol. 43, No. 2, 2007, pp. 290 - 300.

Hegiy, A., L. Mihaylova, R. Boel, and Z. Lendek, Parallelized particle filtering for freeway traffic state tracking, 2007.

Munoz, L., X. S. X. Sun, R. Horowitz, and L. Alvarez, Traffic density estimation with the cell transmission model. Proceedings of the 2003 American Control Conference, Vol. 5, 2003, pp. 3750-3755.

Alvarez-Icaza, L., L. Munoz, X. S. X. Sun, and R. Horowitz, Adaptive observer for traffic density estimation. Proceedings of the 2004 American Control Conference, Vol. 3, No. August 2017, 2004, pp. 2705-2710.

Canudas-De-Wit, C., L. L. Ojeda, and A. Y. Kibangou, Graph constrained-CTM observer design for the Grenoble south ring. IFAC Proceedings Volumes (IFAC-PapersOnline), , No. September 2012, 2012, pp. 197-202.

Vivas, C., S. Siri, A. Ferrara, S. Sacone, G. Cavanna, and F. R. Rubio, Distributed consensus-based switched observers for freeway traffic density estimation. In Proceedings of the IEEE Conference on Decision and Control, 2016, Vol. 2016-Febru, pp. 3445-3450.

Brandi, A., A. Ferrara, S. Sacone, S. Siri, C. Vivas, and F. Rubio, Model predictive control with state estimation for freeway systems. 2017 American Control Conference (ACC), , No. July, 2017, pp. 35363541.

Marinica, N.-E. and R. Boel, Distributed control of urban traffic networks using hybrid models. In Systems and Control, 28th Benelux meeting, Book of abstracts, 2009, pp. 91-91.

Gomes, G. and R. Horowitz, Optimal freeway ramp metering using the asymmetric cell transmission model. Transportation Research Part C, 2006.

Messner, A. and M. Papageorgiou, METANET: A macroscopic simulation program for motorway networks. Traffic Engineering \& Control, 1990.

Payne, H. J., Models of freeway traffic and control. Mathematical models of public systems, 1971.

Spiliopoulou, A., M. Kontorinaki, M. Papageorgiou, and P. Kopelias, Macroscopic traffic flow model validation at congested freeway off-ramp areas. Transportation Research Part C: Emerging Technologies, Vol. 41, 2014, pp. 18-29.

Wang, Y. and M. Papageorgiou, Real-time freeway traffic state estimation based on extended Kalman filter: a general approach. Transportation Research Part B, 2005.

Ngoduy, D., Low Rank Unscented Kalman Filter for Freeway Traffic Estimation Problems. Transportation Research Record, Vol. 5, No. 9, 2011.

Zhang, D., J. Bi, and W. Guan, Freeway Traffic State Estimation Based on Particle Filter. International Journal of Advancements in Computing Technology, Vol. 5, No. 9, 2013. 
Zhang, J. and C. Xia, State-of-charge estimation of valve regulated lead acid battery based on multi-state Unscented Kalman Filter. International Journal of Electrical Power \& Energy Systems, Vol. 33, No. 3, 2011, pp. $472-476$.

Risso, M., A. Rubiales, and P. Lotito, Hybrid method for power system state estimation. IET Generation, Transmission \& Distribution, 2015.

Teixeira, B. O. S., L. A. B. Tôrres, L. A. Aguirre, and D. S. Bernstein, Unscented filtering for intervalconstrained nonlinear systems. Proceedings of the IEEE Conference on Decision and Control, 2008, pp. $5116-5121$.

Valverde, G. and V. Terzija, Unscented Kalman filter for power system dynamic state estimation. IET generation, transmission \& distribution, Vol. 5, No. 1, 2011, pp. 29-37.

Julier, S. J. and J. K. Uhlmann, Unscented filtering and nonlinear estimation. Vol. 92, No. 3, 2004, pp. 401-422.

Van der Merwe, R. and E. A. Wan, The square-root unscented Kalman filter for state and parameterestimation. In Proc. IEEE Int. Conf. Acoustics, Speech, and Signal Processing (ICASSP '01), 2001, Vol. 6, pp. 3461-3464.

Julier, S., J. Uhlmann, and H. F. Durrant-Whyte, A New Method for the Nonlinear Transformation of Means and Covariances in Filters and Estimators. IEEE TRANSACTIONS ON AUTOMATIC CONTROL, Vol. 45, No. 3, 2000, pp. 477-482.

Krajzewicz, D., G. Hertkorn, C. Rössel, and P. Wagner, SUMO-Simulation of Urban MObility-an opensource traffic simulation. In Proceedings of the 4th Middle East Symposium on Simulation and Modelling (MESM20002), 2002, pp. 183-187.

Krauß, S., Microscopic modeling of traffic flow: Investigation of collision free vehicle dynamics. Ph.D. thesis, 1998.

Behrisch, M., L. Bieker, J. Erdmann, and D. Krajzewicz, SUMO - Simulation of Urban MObility. SIMUL 2011 : The Third International Conference on Advances in System Simulation, 2011. 